\title{
Visualization of Collision Behavior of Particles Simulating Inclusions in a Turbulent Molten Steel Flow and Its Theoretical Analysis
}

\author{
Yuji MURAKATA, ${ }^{1)}$ Mun-Gyu SUNG, ${ }^{2)}$ Kensuke SASSA ${ }^{3)}$ and Shigeo ASAI ${ }^{21}$ \\ 1) Graduate Student, Department of Materials Processing Engineering, Graduate School of Engineering, Nagoya University, \\ Furo-cho, Chikusa-ku, Nagoya 464-8603 Japan. $\quad$ 2) Department of Materials Processing Engineering, Graduate School of \\ Engineering, Nagoya University, Furo-cho, Chikusa-ku, Nagoya 464-8603 Japan. $\quad 3$ 3aterials Research Institute for \\ Sustainable Development, National Institute of Advanced Industrial Science and Technology, Anagahora, Shimoshidami, \\ Moriyamaku, Nagoya 463-8560 Japan.
}

(Received on November 6, 2006; accepted on February 21, 2007; originally published in Tetsu-toHagané, Vol. 92, 2006, No. 10, pp. 579-583)

\begin{abstract}
The separation of inclusions with sizes less than several micrometers is very difficult due to their slow terminal velocity. Thus, increasing the volume of inclusion is essential for accelerating the removal of inclusions, and several methods for enhancing collision frequency of inclusions have been proposed, hitherto. Their collision behavior, however, has not been directly observed yet. In this study, by using a water model, the collision rate of polystyrene particles simulating inclusions in a molten steel was quantified by direct observation of the particle collision behavior in a turbulent flow. The collision rate of the particles counted by use of an image analysis method was compared with a theoretical collision rate, which was calculated by substituting the turbulent energy dissipation rate obtained in a numerical analysis into the Saffman-Turner's equation. In the range with large turbulent energy dissipation rate beyond $0.06 \mathrm{~m}^{2} / \mathrm{s}^{3}$, the observed collision rates deviated above the theoretical values. In view of this, the Saffman-Turner's equation has been modified so that it can be applied to the range with large turbulent energy dissipation rate.
\end{abstract}

KEY WORDS: inclusion; clean steel; collision rate; Saffman-Turner's equation; water model experiment; dissipation rate of turbulent energy; Kolmogorov's microscale.

\section{Introduction}

Nonmetallic inclusions such as $\mathrm{Al}_{2} \mathrm{O}_{3}, \mathrm{SiO}_{2}$, etc. produced in deoxidation treatments, degrade the mechanical property of steels so that an effective removal method of inclusions has been desired to produce high quality steels. At present, conventional methods for eliminating inclusions from a molten steel are based on the utilization of gravity force. These methods, however, do not work well for small inclusions less than several micrometers because the terminal velocity of inclusions depends on their volume and it takes rather long time for separation of small inclusions.

As the collision of small inclusions is essential to increase the volume of inclusions, several methods have been proposed, hitherto. ${ }^{1-3)}$ Many hydrodynamic and statistical theories on the collision of inclusions have been propounded $^{4-12)}$ and mathematical analyses for predicting the collision behavior of inclusions in a molten steel have been proposed $^{13-15)}$ so far. The collision behavior, however, has not been directly observed yet even in a water model experiment.

In the present study, based on the numerical calculation of a flow field and the visualization of the collision behavior of polystyrene particles suspended in a water, the
Saffman-Turner's equation describing the collision frequency of inclusions in a turbulent flow has been modified so that it can be applied to the high turbulent flow where the Kolmogoroff's micro-scale becomes as small as a particle size.

\section{Collision Theory of Particles}

The coagulation of inclusions in a fluid flow can be classified according to the type of collisions; collision due to Brownian motion, Stokes motion and turbulent motion. Especially, the collision of particles with small radius in several micrometers is dominated by the collision in a turbulent flow. ${ }^{9)}$ The collision frequency per unit volume in a turbulent flow is expressed by the Saffman-Turner's equation given by Eq. (1), which can hold when a particle radius is far less than the Kolmogoroff's micro-scale, $\eta=\left(v^{3} / \varepsilon\right)^{1 / 4,4)}$

$$
N_{i j}=1.294\left(a_{i}+a_{j}\right)^{3}(\varepsilon / v)^{1 / 2} n_{i} n_{j}, \quad a \ll \eta .
$$

Higashitani et al. ${ }^{6}$ proposed a modified Saffman-Turner's equation as Eq. (2) by taking account of the interaction between Van der Waals and viscous drag forces acting on particles, 


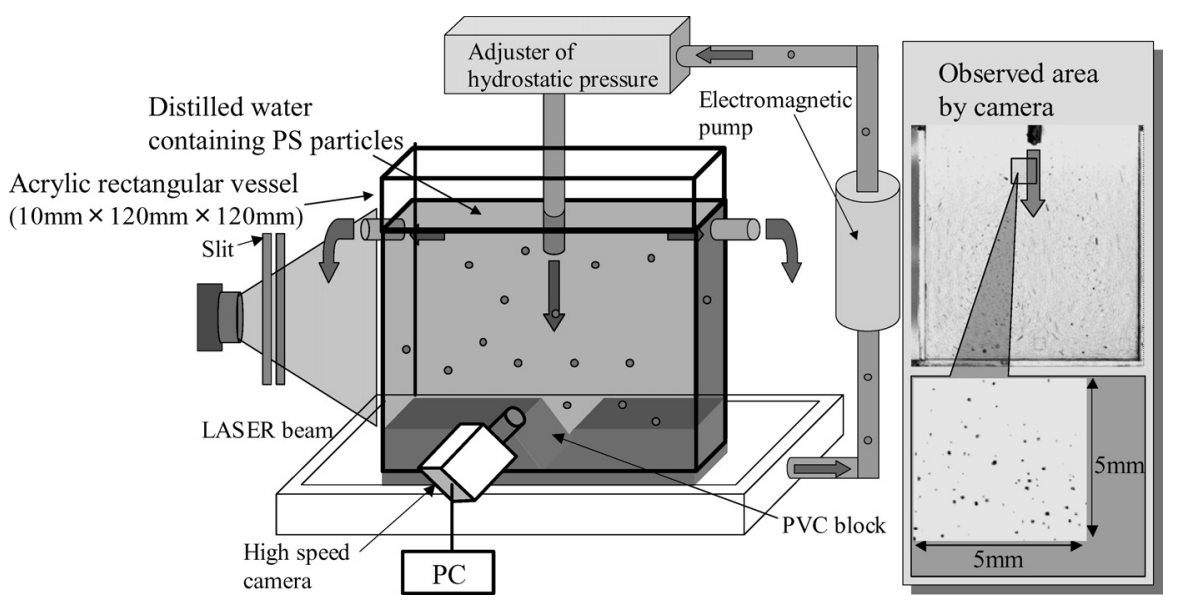

Fig. 1. Schematic view of experimental apparatus.

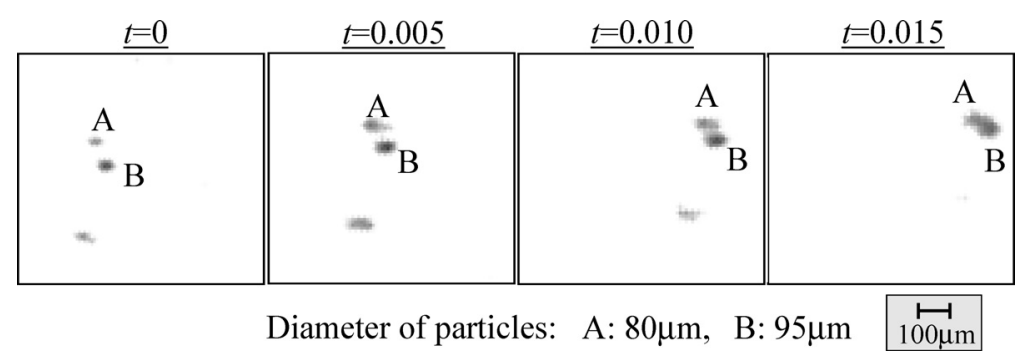

Fig. 2. Collision behavior of particles observed in the model experiment.

$$
N_{i j}=\alpha\left\{1.294\left(a_{i}+a_{j}\right)^{3}(\varepsilon / v)^{1 / 2} n_{i} n_{j}\right\}, \quad a \ll \eta .
$$

where $\alpha$ is the correction coefficient, $a$ the particle radius, $\varepsilon$ the turbulent energy dissipation rate, $v$ the kinetic viscosity coefficient of fluid and $n$ the particle number density.

\section{Experimental}

A water model experiment for simulating the collision behavior of inclusions in a molten steel, was carried out. The experimental apparatus is shown in Fig. 1. An acrylic rectangular vessel with a thickness of $10 \mathrm{~mm}$, a width of $120 \mathrm{~mm}$ and a height of $120 \mathrm{~mm}$ was used. In order to change the fluid velocity and the dissipation rate of turbulent energy in the vessel, three kinds of block were prepared and each of them was set on the bottom of the vessel. Mixed polystyrene particles with two different radii of $23.5 \mu \mathrm{m}$ and $47.5 \mu \mathrm{m}$ or $40 \mu \mathrm{m}$ and $47.5 \mu \mathrm{m}$ with equal particle-number densities were suspended in an aqueous sodium chloride solution, which was used for adjusting the density of particles to the solution. The solution was circulated at the flow velocity by a pump through an inlet and outlet nozzles and the inlet velocity was $0.44 \mathrm{~m} / \mathrm{s}$. The particle behavior was recorded over a local area of $5 \mathrm{~mm} \times 5 \mathrm{~mm}$ in the vessel by a high-speed camera with 1000FPS and the particle collision frequency was counted from the local particle behavior by use of an image analysis method. To observe the particles collision behavior taking place in a two-dimensional plane, a laser slit beam with the thickness of $300 \mu \mathrm{m}$ was inserted from the side of the vessel. In the image analysis, the collision of two particles was detected in the following two cases. One case is when a particle orbit changes due to the interaction of the other particle and the other case is when the images of two particles are overlapped in the direction of the laser slit depth even though the change of the particle orbit is not observed. This criterion could lead to overestimate the collision frequency. The over-counted frequency, however, could not influence analytical results because the criteria of the collision were applied to all the cases and the over-counting could take place in the same probability in all the cases. When the distance between two particles approaches within a distance less than one-tenth or one-fifth of the sum of two particle radii, it has been assumed that the particles have collided though in fact the polystyrene particles never coalesce in a water. Here, the transitional collision behavior of two particles is shown in Fig. 2.

\section{Numerical Analysis}

A three-dimensional numerical calculation on a fluid flow field for the experimental system was carried out for evaluating the turbulent energy dissipation rate at several local positions. The standard $k-\varepsilon$ model was adopted for the calculation. As the boundary conditions, the $1 / 7$ power law was applied as a wall function and the position of a free surface was assumed to be fixed. In addition, the value of 0.4 was adopted as a local maximum Courant number. The distributions of velocity field and turbulent energy dissipation rate in a cross sectional center plane in the depth direction are shown in Figs. 3(a)-3(c) and Figs. 4(a)-4(c), respectively. Figures 4(a) and 4(b) show only the result in the left hand side because of symmetry of the vessel configuration. 


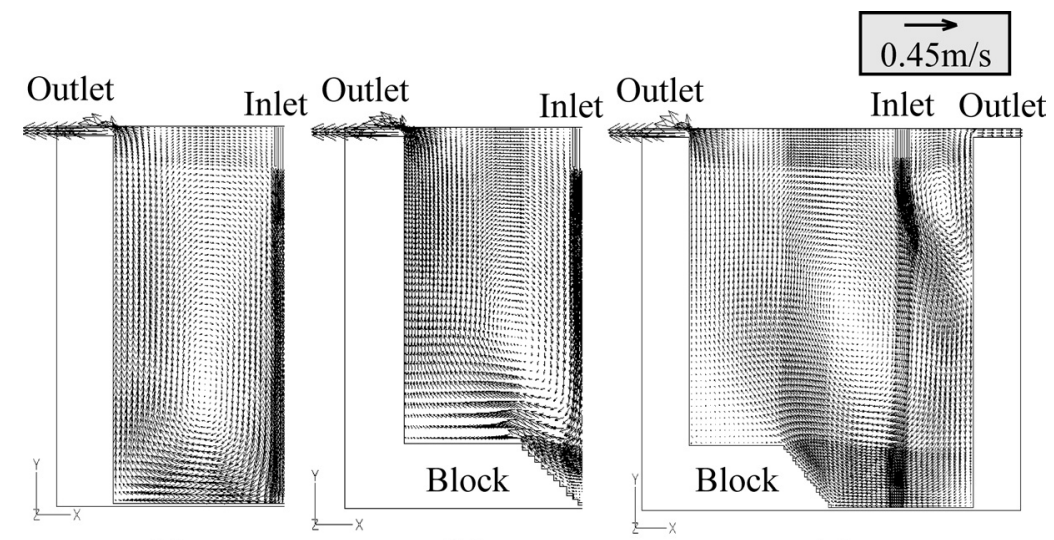

(a)

(b)

(c)

Fig. 3. Distributions of velocity vector.

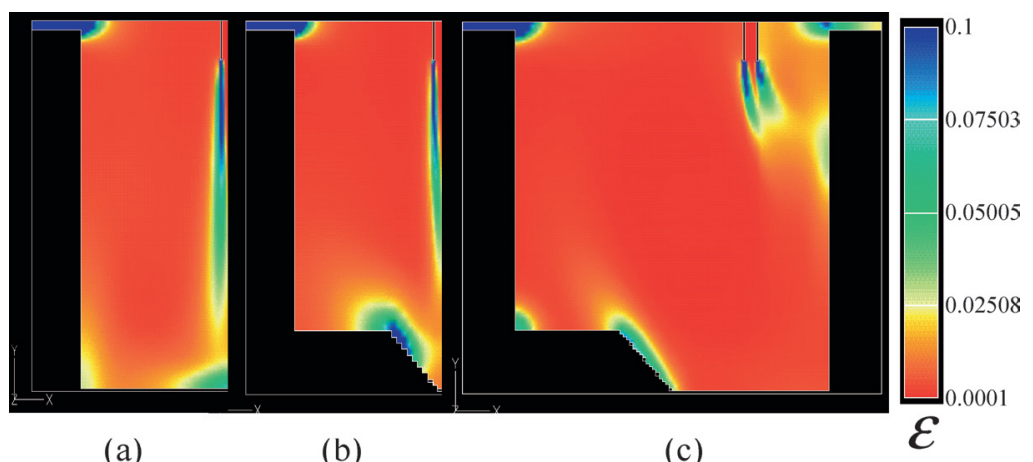

Fig. 4. Distributions of turbulent energy dissipation rate.
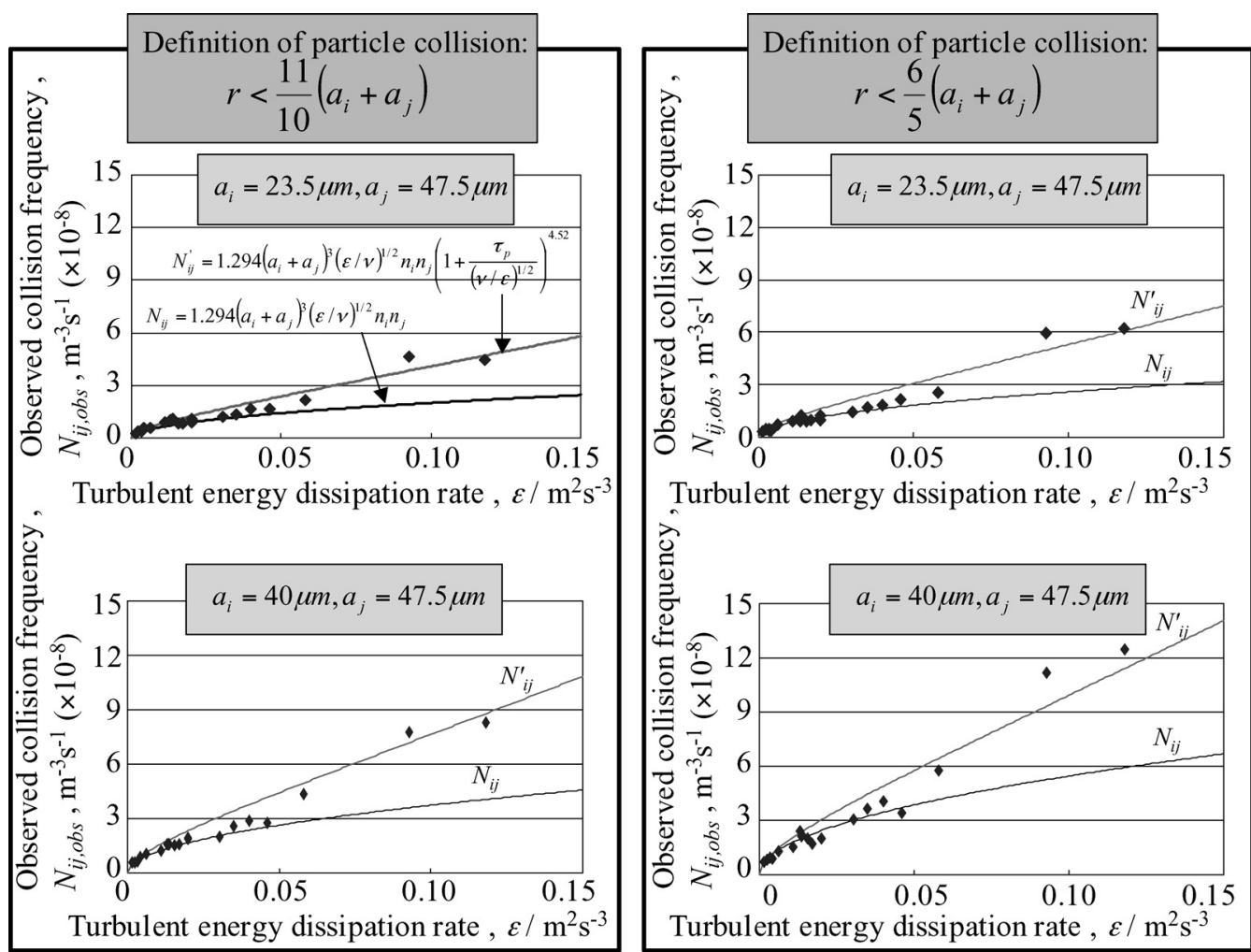

Turbulent energy dissipation rate,$\varepsilon / \mathrm{m}^{2} \mathrm{~s}^{-3}$

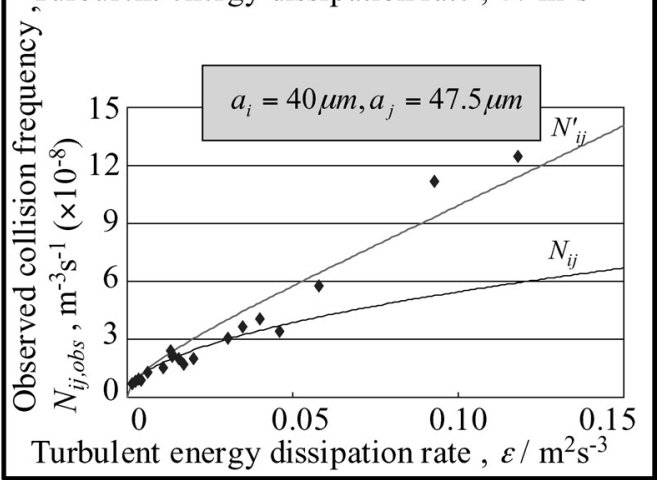

Fig. 5. Comparison between observed collision frequencies and theoretical ones predicted from turbulent energy dissipation rate calculated by use of a fluid flow model.

\section{Results and Discussion}

Figure 5 shows the relationship between the averaged value of particle collision rates obtained in the experiment and those in the numerical analysis, which were calculated by substituting the calculated turbulent energy dissipation 

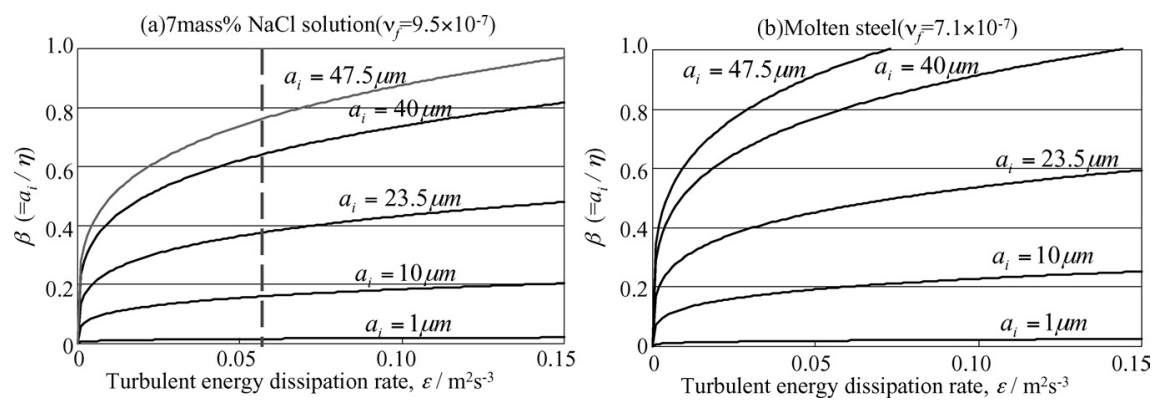

Fig. 6. Relationship between $\beta$ and $\varepsilon$ in various radii of particles.

rates into Eq. (1). As it is not necessary to take account of interaction forces such as Van der Waals and viscosity drag in this experiment, Eq. (1) was used instead of Eq. (2). The solid line indicates the numerically evaluated collision rate. Both the experimental and calculated results agree well with each other in the region where the turbulent energy dissipation rate is less than $0.06 \mathrm{~m}^{2} / \mathrm{s}^{3}$. Though it has been mentioned in the Chap. 3 that the criteria of the collision could lead to overestimate the collision frequency, this agreement tells that the criteria adopted in the experiment is reasonable. On the other hand, the observed particle collision rates are 2-3 times larger than the theoretical prediction in the region where the turbulent energy dissipation rate exceeds $0.06 \mathrm{~m}^{2} / \mathrm{s}^{3}$. This reason can be explained as follows: in the area where the turbulent energy dissipation rate is over $0.06 \mathrm{~m}^{2} / \mathrm{s}^{3}$, particles can not follow the eddy motion and may move irregularly. This irregular motion plays a main role for particle collision.

As mentioned before, Eq. (1) holds only when the collision radius, $a_{i}+a_{j}$, is much smaller than the Kolmogorov's micro-scale, and particles with the radius as large as the Kolmogorov's micro-scale could not follow the eddy motion. That is, in the range of high turbulent energy, the Kolmogorov's micro-scale $\eta$ becomes smaller than the particle size. Here, let us define the ratio $\beta$ of particle radius to Kolmogorov's micro-scale, which is given by the following equation:

$$
\beta \equiv \frac{a_{i}}{\eta}=\frac{a_{i} \varepsilon^{1 / 4}}{v^{3 / 4}}
$$

Figure 6(a) shows the relationship berween $\beta$ and $\varepsilon$ in a water model system having particles with various radii. As mentioned before, $\varepsilon=0.06 \mathrm{~m}^{2} / \mathrm{s}^{3}$ was derived as the position where the observed collision frequencies appear to be larger than the theoretical prediction. In Fig. 6(a) calculated for a water model system, $\beta=0.75$ is figured out for the particle with the radius of $47.5 \mu \mathrm{m}$. Figure 6(b) shows the relationship between $\beta$ and $\varepsilon$ in a molten steel system for different radii of particles. As in practical operations, a molten steel contains inclusions with size distribution from a few to a hundred micrometers, the value of $\beta$ can be estimated to approach to 1 , based on the consideration that the kinematic viscosity coefficient of the molten steel and the order of the turbulent energy dissipation rate in a tundish are almost same as those of the water model system. Therefore, it can be estimated that Eq. (1) does not hold in practical operations. By taking account of the irregular motion of particles, Eq. (1) should be modified as Eq. (4) when a par- ticle radius approaches to the Kolmogorov's micro-scale.

$$
N_{i j}{ }^{\prime}=1.294\left(a_{i}+a_{j}\right)^{3}(\varepsilon / v)^{1 / 2} n_{i} n_{j}\left(1+\frac{\tau_{\mathrm{p}}}{\tau_{\eta}}\right)^{k} \ldots
$$

where $\tau_{\mathrm{p}}\left(=2 a_{i}^{2} \rho_{\mathrm{p}} / 9 \mu\right)$ is the relaxation time of particle motion, $\tau_{\eta}(=\sqrt{\varepsilon / v})$ the extinction time of the Kolmogorov eddy and $k$ the constant. If the size of particles is small and particles follow to the eddy motion entirely, the relaxation time of particle motion is far less than the extinction time of the Kolmogorov eddy so that the value of $\tau_{\mathrm{p}} / \tau_{\eta}$ is negligible in comparison with unit. On the contrary, if the size of particles is same as the Kolmogorov's micro-scale, particles cannot follow the eddy motion. In this case, particles take irregular motion and the value of $\tau_{\mathrm{p}} / \tau_{\eta}$ is not negligible in comparison with unit. Thus, this phenomenon leads to the increase of particle collision frequency. By fitting the experimental data with the theoretical prediction given by Eq. (4), the constant $k$ in Eq. (4) is determined to be 4.52. The curves calculated by use of Eq. (4) are shown in Fig. 5. By adopting the modified formula of Eq. (4), the particle collision frequency in the range of large turbulent energy dissipation rate can be predicted well.

\section{Suggestion for Practical Operation}

If the turbulent energy dissipation rate can be locally increased, the value of $\beta$ also will get to be large at local positions. Thus, the irregular motion of particles increases the particle collision frequency. Therefore, in order to efficiently increase the removal rate of inclusions, at first a molten steel having small inclusions should be so ran into a turbulent region generated under the constraint of limited fluid motion energy as to drastically accelerate the collision of inclusions, and then the molten steel having large inclusions enlarged in the turbulent region should be so brought to a laminar region as to smoothly remove them out of the molten steel.

\section{Conclusions}

To clarify the collision behavior of inclusions in a molten steel, the collision behavior of particles simulating inclusions in a molten steel was directly observed in a water model experiment and the fluid flow field in the model system was calculated for evaluating the turbulent energy dissipation rate. In the area where the turbulent energy dissipation rate is large, the observed collision frequencies were two or three times larger than the theoretical prediction ob- 
tained by substituting the calculated turbulent energy dissipation rate into the Saffman-Turner's equation. The reason is explained as follows: particles cannot follow the eddy motion in the area with large turbulent energy dissipation rate. Based on the observed and calculated collision frequencies of particles, the Saffman-Turner's equation has been modified as:

$$
N_{i j}{ }^{\prime}=1.294\left(a_{i}+a_{j}\right)^{3}(\varepsilon / v)^{1 / 2} n_{i} n_{j}\left(1+\frac{\tau_{\mathrm{p}}}{(v / \varepsilon)^{1 / 2}}\right)^{4.52}
$$

This research was partly supported by the 13th ISIJ Research Promotion Grant, Grant-in-Aid for Scientific Research on Priority Areas (S), number 13852013 from Ministry of Education, Culture, Sports, Science and Technology of Japan, and JSPS Asian Core Program.

\section{Nomenclature}

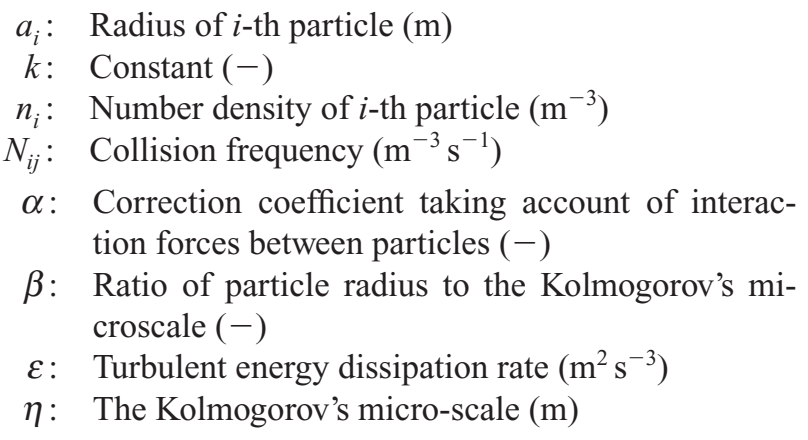

$\mu$ : Viscosity coefficient of fluid $\left(\mathrm{kg} \mathrm{m}^{-1} \mathrm{~s}^{-1}\right)$

$v$ : Kinematic viscosity coefficient of fluid $\left(\mathrm{m}^{2} \mathrm{~s}^{-1}\right)$

$\tau_{\mathrm{p}}:$ Relaxation time of particle motion (s)

$\tau_{\eta}$ : Extinction time of a smallest eddy (s)

$\rho_{\mathrm{f}}$ : Fluid density $\left(\mathrm{kg} \mathrm{m}^{-3}\right)$

$\rho_{\mathrm{p}}$ : Particle density $\left(\mathrm{kg} \mathrm{m}^{-3}\right)$

\section{REFERENCES}

1) K. Okumura, M. Hirasawa, M. Sano and K. Mori: Tetsu-to-Hagané, 80 (1994), 107.

2) S. Hatanaka, T. Taki, M. Kuwabara, M. Sano and S. Asai: Jpn. J. Appl. Phys., 38 (1999), 5B, 3096.

3) T. Kameyama, S. Niwa, J. Park, T. Matumura, K. Sassa and S. Asai: Tetsu-to-Hagané, 89 (2003), 623.

4) P. G. Saffman and J. S. Turner: J. Fluid Mech., 1 (1956), 16.

5) S. Linder: Scand. J. Metall., 3 (1974), 137.

6) K. Higashitani, K. Yamauchi, Y. Matsuno and G. Hosokawa: J. Chem. Eng. Jpn., 16 (1983), 299.

7) S. Taniguchi, A. Kikuchi, T. Ise and N. Shoji: ISIJ Int., 36 (1996), Supplement, S117.

8) H. Tozawa, Y. Kato, K. Sorimachi and T. Nakanishi: ISIJ Int., 39 (1999), 426.

9) H. Lei, L. Wang, Z. Wu and J. Fan: ISIJ Int., 42 (2002), 717.

10) T. Nakaoka, S. Taniguchi, K. Matsumoto and S. T. Johansen: ISIJ Int., 41 (2001), 1103.

11) H. Yin, H. Shibata, T. Emi and M. Suzuki: ISIJ Int., 37 (1997), 946.

12) T. Ishii, N. Kubo, T. K. Bose and M. Iguchi: ISIJ Int., 41 (2001), 1174.

13) D. Mazumdar and R. I. L. Guthrie: ISIJ Int., 39 (1999), 524.

14) J. Zhang and H.-G. Lee: ISIJ Int., 44 (2004), 1629.

15) Q. Hou and Z. Zou: ISIJ Int., 45 (2005), 325. 